\title{
Application and clinical efficacy of modified early unclamping technique in robot-assisted laparoscopic partial nephrectomy
}

\section{Chen Song}

Third Hospital of Hangzhou

\section{Luyao Chen}

The First Affiliated Hospital of Nanchang University

Junhua Li

Third Hospital of Hangzhou

Yanbin Wang ( $745460210 @ q q . c o m$ )

Third Hospital of Hangzhou

\section{Bin Fu}

The First Affiliated Hospital of Nanchang University

\section{Research Article}

Keywords: Modified early unclamping, robotic surgery, partial nephrectomy, renal tumor, renal function

Posted Date: March 3rd, 2022

DOI: https://doi.org/10.21203/rs.3.rs-1109433/v1

License: (c) (1) This work is licensed under a Creative Commons Attribution 4.0 International License. Read Full License 


\section{Abstract}

Objective: To investigate the safety and clinical efficacy of modified early unclamping technique in robotassisted laparoscopic partial nephrectomy (RAPN).

Methods: The clinical data of 38 patients with renal tumor who underwent modified early unclamping technique in RAPN surgery admitted to the Department of Urology, the Third People's Hospital of Hangzhou and the First Affiliated Hospital of Nanchang University from January 2018 to April 2021 were retrospectively analyzed. The control group consisted of 78 patients with renal tumors who underwent standard clamping during the RAPN surgery completed by the same surgeon during the same period. The perioperative related indicators and postoperative renal function recovery were analyzed and compared between the two groups.

Results: All patients $(n=116)$ finished the RAPN successfully, and none was transferred to radical and open surgery in both groups. The warm ischemia time in the modified early unclamping group was significantly lower than that in the standard clamping group $(P<0.001)$. After surgery, the renal function index at each time node in the modified early unclamping group were higher than those in the standard clamping group; renal function gradually returned to near preoperative levels after 3 months in both groups. Postoperative follow-up showed no tumor recurrence or metastasis.

Conclusion: The application of modified early unclamping technique in RAPN surgery is safe and feasible. Compared with standard clamping, modified early unclamping can significantly shorten the warm ischemia time of kidneys, without increasing volume of intraoperative blood loss and complications, which helps to protect the postoperative renal function of patients.

\section{Introduction}

Partial nephrectomy is the surgical modality of choice for the treatment of localized renal tumors measuring less than $7 \mathrm{~cm}$ in size, has the same short-term outcome as radical nephrectomy, preserves more nephrons in the long term and benefits patients more ${ }^{[1]}$. Warm ischemia time is a key factor in partial nephrectomy, and numerous studies have confirmed that warm ischemia time is closely related to postoperative renal function recovery, and the current mainstream view is to recommend controlling warm ischemia time within $30 \mathrm{~min}^{[2]}$. In the minimally invasive era of partial nephrectomy, robotic surgery has been shown to reduce warm ischemia time and reduce volume of intraoperative blood loss compared with laparoscopic surgery. In order to effectively protect the renal function of patients, how to reduce the warm ischemia time is a research focus in recent years ${ }^{[3-5]}$. The concept of early unclamping technique is an innovation proposed by Baumert et al. ${ }^{[6]}$, which can significantly reduce the warm ischemia time of partial nephrectomy, but also brings about an increased risk of intraoperative bleeding. On this basis, we modified and optimized this technique, and this study retrospectively analyzed the clinical data of patients who underwent laparoscopic partial nephrectomy with modified early unclamping technique by a 
single surgeon in different hospitals from January 2018 to April 2021, and compared its clinical efficacy with that of standard clamping technique in the treatment of renal tumors. The report is made as follows.

\section{Materials And Methods}

\subsection{General data}

A total of 116 patients with renal tumor were included in this study. Preoperative contrast-enhanced CT or MRI of the kidneys confirmed the presence of solitary renal tumor without local progression and distant metastasis. All patients underwent robot-assisted laparoscopic partial nephrectomy using Da Vinci surgical system ( $\mathrm{Si}$ or $\mathrm{Xi}$ ) and were divided into two groups according to whether the modified early renal artery unclamping technique was used or not, including 38 patients in the modified early unclamping group and 78 patients in the standard clamping group. There were 23 males and 15 females with mean age of $49.8 \pm 12.3$ years, body mass index of $24.1 \pm 3.2 \mathrm{~kg} / \mathrm{m}^{2}$ and mean tumor size of $4.5 \pm 1.6 \mathrm{~cm}$ in modified early unclamping group; there were 42 males and 36 females with mean age of $51.2 \pm 14.2$ years, body mass index of $23.5 \pm 4.8 \mathrm{~kg} / \mathrm{m}^{2}$ and mean tumor size of $4.3 \pm 1.7 \mathrm{~cm}$ in standard clamping group; there were no statistical differences in general data between the two groups $(P>0.05)$. See Table 1 for details.

\subsection{Surgical methods}

The used surgical approach was the corresponding retroperitoneal or transperitoneal approach according to the size and location of the tumor (dorsal or ventral). After general anesthesia, the lateral decubitus position at the healthy side was taken, and four corresponding trocars were used to puncture the lumbar region (retroperitoneal approach) or abdomen (transperitoneal approach). A pneumoperitoneum pressure was maintained at $12 \mathrm{mmHg}$, and the lens, monopolar electric scissors, and bipolar forceps were placed separately. The renal artery was fully exposed, and the renal tumor was fully dissociated according to the location of the tumor and the need for suture. After clamping the main renal artery with a Bull-dog clip (Figure 1A), the renal parenchyma was opened by monopolar electroshear, and the tumor was completely removed by enucleation push method with the cooperation of an assistant aspirator (Figure 1B). After the bipolar forceps electrocoagulation wound had obvious bleeding points (Figure 1C), the bottom of the wound was continuously sutured with a 3-0 barbed suture (with Hem-o-lok clip on the tail) and the gap of the collecting system was repaired (Figure 1D). After removing the needle, the suture was successively tightened, and the knot-free Hem-o-lok clip was used for fixation to complete the first layer of suture (Figure 1E). In the modified early unclamping group, 2-3 stitches of rapid suture with barbed suture at large spacing between the outer wound edges were continued (Figure 1F), and then the renal artery clamping clip was released to restore the renal blood supply (Figure 1G), followed by continuous suture of the renal wound with barbed suture to anchor the renal parenchyma and keep the wound edges well aligned, and the second layer of suture was completed (Figure 1H). Careful observation was performed on active bleeding and additional needles were inserted if necessary (Figure 1I). In the standard clamping group, the renal artery clamping clip was released after the second-layer suturing was completed to 
restore the renal blood supply. The pararenal drainage tube was indwelled, the specimen was removed by appropriately extending the incision, and the incision was closed layer by layer.

\subsection{Monitoring of renal function parameters}

Serum creatinine was recorded preoperatively, 1 day postoperatively, 1 month postoperatively, and 3 months postoperatively. Renal ECT was also performed at these time points to understand bilateral renal function. This study was approved by the hospital ethics committee with the the patient informed consent provided.

\subsection{Postoperative treatment and follow-up}

According to the treatment method of rehabilitation surgery, the patients were encouraged to get out of bed on the first day after surgery and have fluid diets. The drainage tube was removed after the postoperative drainage volume was $<50 \mathrm{~mL} / \mathrm{d}$, and the patients were discharged on the second day after extubation. Systematic reexamination was performed 3 months after surgery, including blood routine, renal function, glomerular filtration rate GFR, and abdominal and chest CT. Subsequently, regular reexamination and follow-up were performed every 3-6 months to determine whether the tumor had recurrence and metastasis.

\subsection{Statistical methods}

SPSS 19.0 statistical software was used for data analysis. The normally distributed measurement data were expressed as mean \pm standard deviation, and t-test was used; the non-normally distributed measurement data were expressed as median, and non-parametric test was used for intergroup comparison to calculate the differences; the enumeration data were compared by chi-square test across the groups. $P<0.05$ was considered to indicate a statistically significant difference.

\section{Results}

All patients $(n=116)$ underwent the robot-assisted partial nephrectomy successfully, and none was transferred to radical nephrectomy or open surgery in both groups. The comparison of general data and perioperative relevant data between the two groups is shown in Table 1. The differences in the average operation time, postoperative drainage tube removal time and postoperative hospital stay between the two groups had no statistical significance $(P>0.05)$. The warm ischemia time in the modified early unclamping group was significantly lower than that in the standard clamping group (14.8 \pm 3.4 vs $25.5 \pm$ $4.7 \mathrm{~min}, \mathrm{P}<0.001)$. There was no significant difference in the volume of intraoperative blood loss between the modified early unclamping group and the standard clamping group (125.8 \pm 20.5 vs $118.4 \pm$ $21.6 \mathrm{~mL}, \mathrm{P}=0.081$ ). In addition, no intraoperative and postoperative blood transfusion events occurred in the two groups. In terms of complications, no postoperative leakage or rebleeding occurred in the two groups. 
Postoperative pathological results showed the presence of renal tumor, and that there were 79 cases of clear cell carcinoma, 22 cases of angiomyolipoma, 8 cases of papillary renal cell carcinoma, 3 cases of suspicious cell carcinoma and 4 cases of renal cell carcinoma, none of which were positive in resection margin. The patients were followed up for 13.4 months (4-24 months), and no tumor recurrence or metastasis was observed in either group.

There was no significant difference in preoperative renal function index (Serum creatinine, Total GFR and Unilateral GFR) between the two groups; After surgery, the Serum creatinine, Total GFR and Unilateral GFR at each time node in the modified early unclamping group were higher than those in the standard clamping group; renal function gradually returned to near preoperative levels after 3 months in both groups, as shown in Table 2.

\section{Discussion}

With the promotion and popularization of minimally invasive surgery, laparoscopic partial nephrectomy has become the preferred surgical method for T1 renal tumors. The rapid application and development of Da Vinci surgical robots in clinical practice in the past decade has revolutionized surgery. The robotic high-definition 3D stereoscopic field and multi-degree of freedom robotic arm make the operation more precise, especially suitable for the surgery requiring suture reconstruction for partial nephrectomy. A number of domestic and foreign studies have confirmed that in partial nephrectomy (especially for complex and difficult tumors), robotics exerts its significant advantages over laparoscopy ${ }^{[7-10]}$.

The triumph of partial nephrectomy is a goal that urologists continue to pursue, that is, complete resection of the tumor, maximum protection of renal function and no surgical complications. How to protect renal function to the greatest extent has become the focus of attention in partial nephrectomy. On the premise of ensuring negative resection margins, it is important to preserve as much renal parenchyma as possible to protect postoperative renal function. In addition, renal ischemic injury caused by intraoperative renal artery clamping is also one of the important factors causing renal function loss. At present, academia believes that controlling intraoperative warm ischemia time within 30 min or even 25 min is essential for the protection of postoperative renal function.

Based on this, a variety of innovative surgical modes have been proposed to reduce warm ischemia time, including: Renal artery branch clamping technique ${ }^{[11]}$, off-clamp zero-ischemic technique ${ }^{[12]}$ and early unclamping technique ${ }^{[6]}$. However, it is worth noting that the renal artery branch clamping technique requires thorough and sufficient dissociation of the main renal artery and its branches, with high technical difficulty in separation, and branch clamping is also easy to lead to excessive volume of intraoperative blood loss due to incomplete clamping, and even affect the visual field; however, the unclamping technique is more suitable for superficial small volume tumors, and the surgical field is difficult to operate on due to the large amount of bleeding, and predisposes to the formation of positive resection margins. Baumert et al. ${ }^{[6]}$ proposed the concept of early unclamping technique in 2007 , that is, after suturing and closing the inner layer of renal wound under renal artery clamping, relieving the renal 
artery clamping, opening the blood flow and then continuing to suture and close the outer renal wound edge, so as to achieve the purpose of reducing the warm ischemia time; however, there is a risk of increasing intraoperative bleeding.

Several centers in China and abroad have successively reported the clinical application of early unclamping technique. Zhang et al. ${ }^{[13]}$ reported 29 cases of laparoscopic partial nephrectomy with early unclamping. The results showed that the mean warm ischemia time was $13.4 \mathrm{~min}$, significantly lower than $21.1 \mathrm{~min}$ in the control group. San Francisco et al. ${ }^{[14]}$ reported the application of early unclamping technique in robotic surgery, including a total of 12 patients, with an average warm ischemia time of 16 min and a volume of blood loss of $150 \mathrm{ml}$, showing good safety and generalizability. Subsequently, Peyronnet et al. ${ }^{[15]}$ reported 222 large cases of early unclamping-based robot-assisted partial nephrectomy. Compared with the standard clamping group, the early unclamping group had larger and more complex tumor volume. The results showed that the warm ischemia time was shortened by nearly 6 min, but accompanied by more blood loss. The modified early unclamping technique implemented by our center, on the basis of the traditional technique, reduced the warm ischemia time by nearly 7 minutes without increasing the volume of intraoperative blood loss by quickly applying 2-3 stitches at a large distance between the outer layers of the wound before the renal artery unclamping, which fully reflects the technical advantages as well as safety and effectiveness of the modified early unclamping.

Renal artery pseudoaneurysm (RAP) and urine leakage are possible complications after partial nephrectomy. In recent years, many foreign literatures have reported that early unclamping technique has certain advantages for preventing postoperative RAP and urine leakage. Motoyama et al. ${ }^{[16]}$ performed contrast-enhanced CT in 96 patients who underwent robot-assisted partial nephrectomy $3-5$ days after surgery and found that RAP occurred in 7 patients with traditional clamping but not in the early unclamping group. The study performed by Kondo et al. ${ }^{[17]}$ also confirmed that early unclamping technique could reduce the risk of postoperative RAP. Delto et al. ${ }^{[18]}$ showed that early unclamping techniques may reduce the incidence of RAP and urinary leakage by comparing single-center data with data from other centers. One of the important reasons for these advantages is that in the state of open blood flow, the subsequent targeted suture and hemostasis for the wound, especially for inexperienced beginners, are more helpful to reduce the incidence of complications such as postoperative RAP formation, secondary bleeding and urine leakage.

The reduction of warm ischemia time brought about by early unclamping technique has a positive effect on postoperative renal function protection. The data of our center also showed that, the decrease of renal function in the modified early unclamping group was slightly lower than that in the standard clamping group as revealed by the reexamination at 3 months after surgery. The reason for the absence of statistically significant difference in postoperative renal function between the two groups may be attributed to the fact that the preoperative renal function of all enrolled patients was normal, and the overall warm ischemia time of most patients was within 25 minutes. For patients with complex tumors, solitary kidney tumors, or tumors with chronic renal insufficiency, modified early unclamping technique 
significantly reduces warm ischemia time and may have a more significant effect on postoperative renal function protection, which needs to be verified by further studies.

In the traditional early unclamping technique, the inner layer is unclamped after suturing. We found in the clinical practice that, at this time, the renal blood flow was restored, and more bleeding often occurred, making the visual field poor, especially for the deeper and larger wound surface, on which the outer suture tension makes it more difficult after renal congestion. The modified early unclamping technique proposed by us takes only 1 minute to quickly apply 2-3 stitches at large intervals in the outer layer of the wound before the renal artery unclamping, which significantly reduces the wound bleeding after restoring the renal blood flow, and reduces the tension of the subsequent suture. This not only reduces the warm ischemia time, but also compensates for the defects of increased volume of intraoperative blood loss caused by the traditional technique. In recent years, we have completed a certain number of modified early unclamping laparoscopic partial nephrectomies, with some preliminary experience gained: First, secure inner suturing is an important step in surgery. There are many open arterioles or venules in the deep part of the wound after tumor resection. When the first layer of suture is performed, the barbed suture must be "bottomed out" to firmly suture and prevent subsequent excessive bleeding. At the same time, for the occurrence of collecting system damage, the exact closure gap can effectively prevent postoperative leakage; secondly, during the inner layer suture, the needle insertion and needle withdrawal should be appropriately close to the wound edge. After tightening the suture, the wound edge on both sides can be closely aligned; otherwise the congestion and tension of the renal parenchyma are too large after opening the blood flow, which is not conducive to subsequent suture. In addition, this modified technique is especially suitable for deeper and larger complex types of tumors, which can significantly reduce warm ischemia time and oozing after open blood flow, making the operation more easy to complete and more beneficial to patients.

\section{Conclusions}

In summary, the modified early unclamping technique is safe and practical in robot-assisted partial nephrectomy. Compared with the traditional clamping, the modified early unclamping can significantly reduce the warm ischemia time of the kidney without increasing the volume of intraoperative blood loss, which is of great help to the protection of postoperative renal function in patients and has a good value of clinical promotion.

\section{Abbreviations}

RAPN=Robot-assisted laparoscopic partial nephrectomy

ECT=Emission Computed Tomography

GFR=Glomerular Filtration Rate

CT=Computed Tomography 
MRI=Magnetic Resonance Imaging

MDRD=Modification of Diet and Renal Disease

3D=Three-dimensional

RAP=Renal artery pseudoaneurysm

\section{Declarations}

\section{Ethics approval and consent to participate}

The studies involving human participants were reviewed and approved by the Ethics Committee of the Third Hospital of Hangzhou and the First Affiliated Hospital of Nanchang University. The patients/participants provided their written informed consent to participate in this study. All methods were performed in accordance with the relevant guidelines and regulations.

Consent for publication $\square$ Not applicable

\section{Availability of data and materials}

The datasets used and/or analysed during the current study are available from the corresponding author on reasonable request.

Competing interests[The authors declare that they have no competing interests

\section{Funding}

This study was funded by Medical and health science and Technology project of Zhejiang Province (Grant Number. 2022491213) and Zhejiang Province Traditional Chinese Medicine Science and Technology Project (Grant Number. 2021ZB226)

\section{Authors' contributions}

Conception and design: Bin Fu and Chen Song. Acquisition of data: Luyao Chen, Yanbin Wang, and Junhua Li. Analysis and interpretation of data: Yanbin Wang, and Chen Song. Statistical analysis: Junhua Li. Manuscript writing: Luyao Chen, and Junhua Li. Manuscript editing: Bin Fu

\section{Acknowledgements}

The authors thank all the people who support investigators to complete this study.

\section{Data availability statement}

The datasets generated and/or analysed during the current study are not publicly available due the data also forms part of an ongoing study, but are available from the corresponding author on reasonable 
request.

\section{References}

1. U. Capitanio, C. Terrone, A. Antonelli, et al. Nephron-sparing techniques independently decrease the risk of cardiovascular events relative to radical nephrectomy in patients with a T1a-T1b renal mass and normal preoperative renal function[J]. Eur Urol, 2015, 67(4): 683-689.

2. M. N. Simmons, M. J. Schreiber, I. S. Gill. Surgical renal ischemia: a contemporary overview[J]. J Urol, 2008, 180(1): 19-30.

3. McClintock T R, Bjurlin M A, Wysock J S, et al. Can selective arterial clamping with fluorescence imaging preserve kidney function during robotic partial nephrectomy?[J]. Urology, 2014,84(2):327332.

4. Mattevi D, Luciani L G, Mantovani W, et al. Fluorescence-guided selective arterial clamping during RAPN provides better early functional outcomes based on renal scan compared to standard clamping[J]. J Robot Surg, 2019,13(3):391-396.

5. T. Klatte, V. Ficarra, C. Gratzke, et al. A Literature Review of Renal Surgical Anatomy and Surgical Strategies for Partial Nephrectomy[J]. Eur Urol, 2015, 68(6): 980-992.

6. H. Baumert, A. Ballaro, N. Shah, et al. Reducing warm ischaemia time during laparoscopic partial nephrectomy: a prospective comparison of two renal closure techniques[J]. Eur Urol, 2007, 52(4): 1164-1169.

7. Hinata N, Shiroki R, Tanabe K, et al. Robot-assisted partial nephrectomy versus standard laparoscopic partial nephrectomy for renal hilar tumor: A prospective multi-institutional study[J]. Int $\mathrm{J}$ Urol, 2021,28(4):382-389.

8. Kwon K J, Ryu H, Kim M, et al. Personalised three-dimensional printed transparent kidney model for robot-assisted partial nephrectomy in patients with complex renal tumours (R.E.N.A.L. nephrometry score >/=7): a prospective case-matched study[J]. BJU Int, 2020.

9. Mita K, Nomura N, Murata D, et al. [Robot-Assisted Partial Nephrectomy for Renal Tumor $</=7 \mathrm{~cm}$ in Diameter][J]. Hinyokika Kiyo, 2018,64(12):477-482.

10. L. Gu, X. Ma, B. Wang, et al. Laparoscopic vs robot-assisted partial nephrectomy for renal tumours of $>4 \mathrm{~cm}$ : a propensity score-based analysis[J]. BJU Int, 2018, 122(3): 449-455.

11. P. Shao, C. Qin, C. Yin, et al. Laparoscopic partial nephrectomy with segmental renal artery clamping: technique and clinical outcomes[J]. Eur Urol, 2011, 59(5): 849-855.

12. I. S. Gill, M. S. Eisenberg, M. Aron, et al. "Zero ischemia" partial nephrectomy: novel laparoscopic and robotic technique[J]. Eur Urol, 2011, 59(1): 128-134.

13. T. Zhang, L. Zhao, J. Ma, et al. Early Unclamping Laparoscopic Partial Nephrectomy for Complex Renal Tumor: Data from a Chinese Cohort[J]. Urol Int, 2019, 102(4): 399-405.

14. I. F. San Francisco, M. C. Sweeney, A. A. Wagner. Robot-assisted partial nephrectomy: early unclamping technique[J]. J Endourol, 2011, 25(2): 305-308. 
15. B. Peyronnet, H. Baumert, R. Mathieu, et al. Early unclamping technique during robot-assisted laparoscopic partial nephrectomy can minimise warm ischaemia without increasing morbidity[J]. BJU Int, 2014, 114(5): 741-747.

16. D. Motoyama, Y. Matsushita, H. Watanabe, et al. Improved perioperative outcomes by early unclamping prior to renorrhaphy compared with conventional clamping during robot-assisted partial nephrectomy: a propensity score matching analysis[J]. J Robot Surg, 2020, 14(1): 47-53.

17. T. Kondo, T. Takagi, S. Morita, et al. Early unclamping might reduce the risk of renal artery pseudoaneurysm after robot-assisted laparoscopic partial nephrectomy[J]. Int J Urol, 2015, 22(12): 1096-1102.

18. J. C. Delto, P. Chang, S. Hyde, et al. Reducing Pseudoaneurysm and Urine Leak After Robotic Partial Nephrectomy: Results Using the Early Unclamping Technique[J]. Urology, 2019, 132: 130-135.

\section{Tables}

Table 1

Comparison of clinical data between modified early unclamping and standard clamping in robot-assisted partial nephrectomy (Mean \pm SD)

\begin{tabular}{|llll|}
\hline \multirow{2}{*}{ Item } & $\begin{array}{l}\text { Modified early unclamping } \\
\text { group }\end{array}$ & $\begin{array}{l}\text { Standard clamping } \\
\text { group }\end{array}$ & $\begin{array}{l}\text { P } \\
\text { value }\end{array}$ \\
\cline { 2 - 4 } & $\mathbf{N = 3 8}$ & $\mathbf{N}=\mathbf{7 8}$ & \\
\hline Gender (male/female) & $23 / 15$ & $42 / 36$ & 0.496 \\
\hline Age/years & $49.8 \pm 12.3$ & $51.2 \pm 14.2$ & 0.604 \\
\hline Body mass index/kg/m 2 & $24.1 \pm 3.2$ & $23.5 \pm 4.8$ & 0.487 \\
\hline Tumor size/cm & $4.5 \pm 1.6$ & $4.3 \pm 1.7$ & 0.546 \\
\hline Operation duration/min & $125.3 \pm 21.6$ & $117.6 \pm 24.9$ & 0.106 \\
\hline Warm ischemia time/min & $14.8 \pm 3.4$ & $25.5 \pm 4.7$ & 0.001 \\
\hline $\begin{array}{l}\text { Volume of intraoperative blood } \\
\text { loss/ml }\end{array}$ & $125.8 \pm 20.5$ & $118.4 \pm 21.6$ & 0.081 \\
\hline $\begin{array}{l}\text { Postoperative drainage tube removal } \\
\text { time/day }\end{array}$ & $4.2 \pm 0.5$ & $3.9 \pm 0.9$ & 0.058 \\
\hline Postoperative hospital stay/day & $5.3 \pm 0.6$ & $5.4 \pm 0.9$ & 0.536 \\
\hline
\end{tabular}

Table 2. Changes of renal function in patients receiving modified early unclamping and standard clamping group in robot-assisted partial nephrectomy (Mean \pm SD) 


\begin{tabular}{|lcll|}
\hline Group & $\begin{array}{c}\text { Serum creatinine } \\
(\mu \mathrm{mol} / \mathrm{L})\end{array}$ & $\begin{array}{c}\text { Total GFR } \\
\nabla \mathrm{mL} / \mathrm{min} \rrbracket\end{array}$ & Unilateral GFR $\rrbracket \mathrm{mL} / \mathrm{min} \rrbracket$ \\
\hline Modified early unclamping group $(\mathrm{n}=38)$ & & \\
\hline Preoperation & $74.5 \pm 4.3$ & $76.4 \pm 7.8$ & $33.6 \pm 4.5$ \\
\hline 1 day postoperation & $90.9 \pm 2.7^{\#}$ & $52.5 \pm 6.1^{* \#}$ & $25.1 \pm 3.2^{\#}$ \\
\hline 1 month postoperation & $85.6 \pm 7.6^{\#}$ & $70.2 \pm 5.7^{\#}$ & $31.1 \pm 2.3^{\#}$ \\
\hline 3 months postoperation & $73.3 \pm 4.8^{\#}$ & $73.1 \pm 7.9^{\#}$ & $32.2 \pm 1.9^{\#}$ \\
\hline Standard clamping group $(\mathrm{n}=78)$ & & \\
\hline Preoperation & $73.3 \pm 5.6$ & $75.8 \pm 6.9$ & $32.8 \pm 3.8$ \\
\hline 1 day postoperation & $101.5 \pm 3.8^{*}$ & $43.4 \pm 3.6^{*}$ & $18.2 \pm 2.6$ \\
\hline 1 month postoperation & $90.3 \pm 3.4^{*}$ & $53.1 \pm 4.3^{*}$ & $19.4 \pm 2.1$ \\
\hline 3 months postoperation & $82.7 \pm 7.9^{*}$ & $52.6 \pm 3.5^{*}$ & $21.3 \pm 1.9$ \\
\hline
\end{tabular}

Note: * vs Preoperation, $P<0.05$; \# vs Standard clamping group, $P<0.05$.

\section{Figures}

\section{Figure 1}

Surgical process of modified early unclamping technique in robot-assisted partial nephrectomy. A. Dissociate the main renal artery and clamp with Bull-dog clamp; B. Completely remove the renal tumor with enucleation push method; $C$. Examine the wound surface and electrocoagulation with bipolar forceps for obvious bleeding points; D. Suture the inner wound with barbed suture; E. Tighten the inner suture and fix with Hem-o-lok clamp; F. Quickly suture the outer layer of barbed suture with large spacing for 2-3 stitches; G. Early unclamping of renal artery to restore the renal blood supply; $\mathrm{H}$. Continue to align the outer wound edge with barbed suture; I. Complete the outer suture to observe whether there is active bleeding, and apply additional needles when necessary. 